\title{
Vegetarian Diets for Weight Loss: How Strong is the Evidence?
}

\author{
Wendy L. Bennett, MD, MPH ${ }^{1,2}$ and Lawrence J. Appel, MD, MPH ${ }^{1,2}$ \\ 'Department of Medicine, Division of General Internal Medicine, The Johns Hopkins University School of Medicine, Baltimore, MD, USA; ${ }^{2}$ Welch \\ Center for Prevention, Epidemiology and Clinical Research, The Johns Hopkins University, Baltimore, MD, USA.
}

J Gen Intern Med 31(1):9-10

DOI: $10.1007 / \mathrm{s} 11606-015-3471-7$

(c) Society of General Internal Medicine 2015

$\mathrm{V}$ egetarian diets are common, well-accepted dietary patterns that are increasingly recommended as healthy options by professional organizations ${ }^{1}$ and nutrition policy makers. ${ }^{2}$ In vegetarian diets, meat-free protein sources include legumes, nuts, and grains, and-in lacto-ovo-vegetarian diets - dairy products and eggs. Reduced meat and vegetarian diets have been associated with a multitude of health benefits, including lower all-cause and cancer-specific mortality, reduced blood pressure and reduced risk of type 2 diabetes. ${ }^{3-6}$ Whether elimination of meat and its substitution with other foods (e.g. fiber rich plant foods) affect weight or accomplish weight loss is less certain.

In this issue of the Journal of General Internal Medicine, Huang and colleagues conducted a systematic review and meta-analysis to assess the effects of vegetarian diets on weight loss. ${ }^{7}$ They identified 12 randomized trials with a total of 1151 participants (range: 11 to 291 per trial) that compared vegan diets (eight studies) or lacto-ovo diets (four studies) to non-vegetarian diets and that included body weight as a study outcome. Median study duration was only 18 weeks (range: 8 weeks to 2 years). Overall, participants in the vegetarian diet arms lost $2 \mathrm{~kg}$ more weight than those in the non-vegetarian diet arms. The mean difference in weight loss was slightly greater for those in the vegan $(2.5 \mathrm{~kg})$ compared with the lactoovo $(1.5 \mathrm{~kg})$ diet arms.

The majority of included studies were deemed low quality and had heterogeneous methods, particularly with respect to characteristics of the randomized groups and the types of diets in the intervention and control arms. The highest quality study was a 12-month randomized trial that compared four popular diets-Atkins, Zone, Ornish and Weight Watchers-in 160 overweight or obese adults. ${ }^{8}$ The group randomized to the Ornish diet, a low-fat vegetarian diet, had the lowest adherence (only $50 \%$ compared to $65 \%$ for Weight Watchers) and achieved non-significantly greater weight loss at 12 months ( 3.3 vs. 2.1 to $3.2 \mathrm{~kg}$ for other diets). ${ }^{8}$ In Huang's metaanalysis, ${ }^{7}$ the longest study lasted 24 months, assessed weight loss maintenance for a vegan vs. a moderate low-fat diet (from

Published online July 22, 2015
National Cholesterol Education Program), and showed significantly greater weight loss with the vegan diet at 1 year ( $4.9 \mathrm{vs}$. $1.8 \mathrm{~kg}$ ) and 2 years ( 3.1 vs. $0.8 \mathrm{~kg})$, with similar adherence rates between the two diets. ${ }^{9}$ However, because few studies lasted longer than 6 months, this review was not able to assess the effects of vegetarian diets on long-term weight loss or maintenance.

The systematic review conducted by Huang and colleagues is notable because it is the first to focus on the effects of vegetarian diets on the outcome of weight loss. ${ }^{7}$ Another recently published systematic review used network meta-analysis to compare weight loss outcomes in low-carbohydrate diets (such as Atkins and Zone), low-calorie diets (such as Weight Watchers) and lowfat diets (such as Ornish), but did not classify diets as vegetarian/vegan vs. non-vegetarian. The network meta analytic approach permitted comparisons of each diet against each other and against no diet; compared to no diet, weight loss was $\sim 8 \mathrm{~kg}$ at 6 months and $\sim 6 \mathrm{~kg}$ at 12 months for the various diets, with a similar effect for all diets, including the low-fat Ornish (vegetarian diet). ${ }^{10}$ Still, because interventions in weight loss trials are commonly multi-factorial and promote calorie reduction together with advice on diet pattern (e.g., low fat, low carbohydrate, or vegetarian), it is difficult to tease apart the effects of a specific diet from behavioral changes (e.g., portion control and tracking calories), which also vary by trial and by randomized arm. With this in mind, it is noteworthy that half of the trials implemented 'energy restriction' with vegetarian diets; these trial achieved a greater mean reduction in weight loss compared to vegetarian diet interventions without energy restriction $(-2.2 \mathrm{~kg}$ vs. $-1.6 \mathrm{~kg})$.

Overall, the study by Huang and colleagues suggests that adoption of a vegetarian diet might accomplish modest weight loss in persons who are overweight or obese. ${ }^{7}$ However, a meta-analysis is only as strong as the contributing trials. In this case, as in numerous other meta-analyses, the evidence base is relatively weak - just 12 trials, the majority of which were small in size and short in duration. Still, the study could be useful for weight loss counseling. A vegetarian diet joins the extensive list of other diets that have modest effects on weight and could be an appealing option for many people because of other healthful benefits or personal preferences. Nonetheless, regardless of the diet that 
patients consume, reduced calorie intake remains the bedrock of behavioral weight loss interventions.

Conflict of interest: The authors do not have any conflicts of interest.

Corresponding Author: Wendy L. Bennett, MD, MPH; Department of Medicine, Division of General Internal MedicineThe Johns Hopkins University School of Medicine, 2024 E. Monument St, Suite 2-616, Baltimore, MD 21205, USA (e-mail: wendy.bennett@jhmi.edu).

\section{REFERENCES}

1. Craig WJ, Mangels AR. American Dietetic Association. Position of the American Dietetic Association: Vegetarian diets. J Am Diet Assoc. 2009; 109(7): 1266-82.

2. U.S. Department of Agriculture and U.S. Department of Health and Human Services. Dietary Guidelines for Americans. 7th ed. Washington: US Government Printing Office; 2010.
3. Yokoyama Y, Nishimura K, Barnard ND, et al. Vegetarian diets and blood pressure: A meta-analysis. JAMA Intern Med. 2014;174(4):577-87.

4. Aune D, Ursin G, Veierod MB. Meat consumption and the risk of type 2 diabetes: A systematic review and meta-analysis of cohort studies. Diabetologia. 2009;52(11):2277-87.

5. Sinha R, Cross AJ, Graubard BI, Leitzmann MF, Schatzkin A. Meat intake and mortality: A prospective study of over half a million people. Arch Intern Med. 2009;169(6):562-71.

6. Orlich MJ, Singh PN, Sabate J, et al. Vegetarian dietary patterns and the risk of colorectal cancers. JAMA Intern Med. 2015;175(5):767-76.

7. Huang RY, Huang CC, Hu FB, Chavarro J. Vegetarian diets and weight reduction: A meta-analysis of randomized controlled trials. J Gen Intern Med. 2015; SPI 3390.

8. Dansinger ML, Tatsioni A, Wong JB, Chung M, Balk EM. Meta-analysis: The effect of dietary counseling for weight loss. Ann Intern Med. 2007; 147(1):41-50.

9. Turner-McGrievy GM, Barnard ND, Scialli AR. A two-year randomized weight loss trial comparing a vegan diet to a more moderate low-fat diet. Obesity (Silver Spring). 2007;15(9):2276-81.

10. Johnston BC, Kanters S, Bandayrel $\mathbf{K}$, et al. Comparison of weight loss among named diet programs in overweight and obese adults: A metaanalysis. JAMA. 2014;312(9):923-33. 